International Review of Research in Open and Distributed Learning Volume 21, Number 2

April - 2020

\title{
Editorial - Volume 21, Issue 2
}

Dietmar Kennepohl

Interim Co-editor, Athabasca University

Welcome to the second issue of 2020. The sudden worldwide appearance of COVID-19 has captured the attention and energy of almost every aspect of society including how we view and approach open and distributed learning. Indeed, many educational jurisdictions have outright cancelled all in-person classes (K-12 and post-secondary) and are now looking to move to remote learning to temporarily bridge the gap. I and many of my open university colleagues have been approached by desperate educators wanting to immediately convert their courses right in the middle of term. Even with over 25 years of online and distance experience, this is new territory for me. I am used to developing courses and then running them. Moving over from in-person to remote during the course itself is a bit like trying to change the tire on a car while it is still driving down the highway. However, it will get done, because it needs to be done. If you were ever looking for motivational opportunity to convince fellow educators, overly comfortable and set in the in-person mode of teaching, to look at alternative teaching modes-this is it. Never waste a crisis.

I fully expect that in upcoming issues of IRRODL we will see studies relating and exploring some of these experiences from around the globe. Essentially, what can we learn from all this? In this respect, I take a more positive view in the long run. There will, of course, be practical lessons learned like moving the last month of term lectures and assignments online, setting up alternate laboratory experiences, running remote examinations, and hopefully some of those will be more than slapped-on solutions like long lecture recordings and textbook dumps. However, there will also be some bigger overarching lessons. One of the more important ones for me is the realization that despite the commonly accepted emphasis on the individual, we are still all very much connected with and responsible for each other worldwide. I believe this is something that naturally resonates with researchers in an area with a strong social mandate like open and distributed learning.

On a side note, you already know that IRRODL currently requires APA Style (sixth edition) for its submissions. A new Publication Manual (seventh edition) was released in October 2019 and in the coming months we plan to transition from the sixth to seventh edition style. Keep posted for details and the exact date for this upcoming changeover. In the meantime, please continue to use APA Style (sixth edition).

In our first paper of this issue Zapata explores the development of instructional modules for an open textbook in a "book sprint" fashion by graduate students. While there were several tangible benefits identified, analysis indicated some gaps in the materials produced, which could have been a consequence of the structure of the book sprint itself. 
Through their exploratory qualitative study of online postgraduates, Peacock, Cowan, Irvine, and Williams identify and discuss three important themes (interaction/ engagement, the culture of the learning, and support) in promoting a sense of belonging and in ensuring that there are opportunities for meaningful group and peer interactions.

In the following study, Rushidi, Shishi Kumar, Yon Rosli, and Baderisang provide insight into the factors that may strengthen relationships and enable universities to increase both their service recovery satisfaction level and overall student retention rates. The findings of this study also confirm and highlight the influence of service recovery satisfaction on behavioural outcomes, which could inform future strategies to encourage learner loyalty and persistence.

Despite cost savings and demonstrated effectiveness of open textbooks, perceptions about quality, relevance, ease of access, and other concerns persist. Bethel confirms the quality measure developed for post-secondary study in the Bahamas as a viable practical tool to assess open resources and found that the open textbooks studied were accessible and well matched to course content, but of varying quality.

Teaching practice is a significant practical component of pre-service teacher education programs even at a virtual university. Abdullah and Mirza investigate the quality of these modules that train the prospective teachers coming from an online educational setting for their professional teaching careers.

In the next article, AlDahdouh examines the prevalence and distribution of learner emotions in connectivist environments. Implications for researchers and teachers in distance education are discussed including that negative-activating emotions can somehow produce positive consequences-although not always.

Andoh, Appiah, and Agyei explore perceptions of postgraduate distance education students which revealed that was not dependent on age, gender, or programme of study, but was significantly related to study centre location and semester of study. Furthermore, the authors critically consider and discuss learner views on physical facilities, staff-students relationship, facilitator quality, and student support services.

University teachers are the prime movers for integrating e-learning systems into higher education institutions and therefore understanding their willingness to use such technology is critical. In this technology acceptance study Goh, Hii, Tan, and Rasli advance our current understanding by identifying the dual roles of motivation for instrumental use, as both cause and mediator.

One final word. Because of the heightened awareness of open and distributed learning it might be a good opportunity to make interested colleagues aware of IRRODL. It is available free-of-charge to anyone and is a tremendous resource for researcher and practitioner alike. 
Editorial - Volume 21, Issue 2

Kennepohl

Athabasca

University

(c) (i) 